\title{
A Note on Pivotality
}

\author{
Addison Pan 1
}

Department of Economics, University of Auckland Business School, Sir Owen G Glenn Building, 12 Grafton Rd, Auckland 1010, New Zealand; addison.pan@auckland.ac.nz; Tel.: +64-9-923-2406

Received: 4 April 2019; Accepted: 29 May 2019; Published: 1 June 2019

\begin{abstract}
This note provides simple derivations of the equilibrium conditions for different voting games with incomplete information. In the standard voting game à la Austen-Smith and Banks (1996), voters update their beliefs, and, conditional on their being pivotal, cast their votes. However, in voting games such as those of Ellis (2016) and Fabrizi, Lippert, Pan, and Ryan (2019), given a closed and convex set of priors, ambiguity-averse voters would select a prior from this set in a strategy-contingent manner. As a consequence, both the pivotal and non-pivotal events matter to voters when deciding their votes. In this note, I demonstrate that for ambiguous voting games the conditional probability of being pivotal alone is no longer sufficient to determine voters' best responses.
\end{abstract}

Keywords: jury trial; pivotality; ambiguity

JEL Classification: D71; D72; D81

\section{Introduction}

Since Austen-Smith and Banks [1] pointed out that sincere voting may not constitute a Nash Equilibrium of a voting game with incomplete information, a series of papers and startling results based on their basic model have followed. This is so, in spite of the many criticisms surrounding the assumptions of their model provided, for instance, by Margolis [2-4] and Dietrich and Spiekermann $[5,6]$. The main critique of these authors is the blunt application of game theory to political science: Since these results rely on the pivotality condition, the results so obtained are, simply put, unrealistic and too surprising to be useful to predict real-world behaviour. The idea that voters determine their votes only by conditioning on them being pivotal, i.e., in the event that they affect the voting outcome, is considered to be too reductive of what drives voting behaviour.

However, this is not as heroic as it is accused of being as an equilibrium condition in a normative sense when strategic voting akin to that defined by Austen-Smith and Banks [1] is considered. It remains to be proved whether pivotality is a necessary and sufficient condition for Bayesian Nash Equilibria of other strategic voting games. That is, whether, in the presence of ambiguity, the equilibrium also depends on the evaluation of events in which the voter is not pivotal.

In essence, the establishment of the pivotal logic is a result of the expected utility assumption in instrumental voting models, inter alia, those of Austen-Smith and Banks [1] and Feddersen and Pesendorfer [7]. In these models, instrumental voters determine their votes, conditional on the probability that their votes affect the final outcome of the voting game. Voters compute the expected payoffs of different actions and define their best responses.

In the model of Austen-Smith and Banks [1], the instrumental voters have a single common prior. Hence, the probability of each non-pivotal event is a singleton and is act-independent. As a consequence of the expected utility assumption, when a voter is confronted with such events, not only their vote does not affect the voting outcome, but also the expected utility under non-pivotality cancels out. As a consequence, the only remaining component that matters is when the voter is pivotal. 
This implies that the pivotality condition is a necessary and sufficient condition for the Bayesian Nash voting equilibria to be established.

Conversely, in voting games with subjective uncertainty, such as those of Ellis [8] and Fabrizi et al. [9], given a closed and convex set of priors, ambiguity-averse voters would select a prior from this set in a strategy-contingent manner. As a result, although voters' strategies do not change their pay-offs in non-pivotal states, they affect their subjective beliefs with respect to the relevant states. Hence, the expected utility under non-pivotality need not cancel out. Instead, voters update their beliefs, and conditionally both on their being pivotal and non-pivotal, cast their votes. In the model of Ellis [8], voters have the Maxmin preferences as in [10]. Thus, they prefer randomising to hedge the subjective uncertainty. This results in the failure of information aggregation in collective voting under ambiguity.

In this note, I provide a more explicit proof for the equilibrium condition for a general common-value voting model under ambiguity. I give a more detailed proof than Austen-Smith and Banks [1] did in their appendix. With an extended set of notations, I define general voting games under ambiguity. I check the equilibrium conditions for voting games with ambiguity such as those in Ellis [8]. Additionally, I generalise the results of Ellis [8] to the case with ambiguity about the conditional distribution of signals and illustrate the equilibrium conditions for voting games such as Fabrizi et al.'s [9].

In contrast to Austen-Smith and Banks [1], in the latter models, I show that the pivotality condition alone is not sufficient to determine voters' best responses. Hence, even in an instrumental voting setup, the relaxation of the expected utility assumption-for instance, when voters are Maxmin expected utility maximisers under a close and convex set of priors-makes a prominent difference in terms of how voters determine their voting behaviours.

Besides Ellis [8] and Fabrizi et al. [9], there are several other authors discussing the modification of the pivotal logic beyond the expected utility, even when maintaining the pivotal voter assumption. Respectively, Eliaz, Ray, and Razin [11] and Dillenberger and Raymond [12] relaxed the expected utility assumption by allowing voters to have rank-dependent preferences (as in Quiggin [13]) and quasi-convex preferences. These two studies not only provide explanations for the choice shifts observed in the group voting context but also reveal that voters may base their decisions on non-pivotal events.

This note proceeds as follows. In Section 2, I carefully derive pivotality conditions for voting games as those considered by Austen-Smith and Banks [1]. In Section 3, I define the ambiguous voting game and show the equilibrium condition for it. I also use the models of Ellis [8] and Fabrizi et al. [9] to illustrate the insufficiency of conditioning merely on pivotality. The last section concludes.

\section{Pivotality in Austen-Smith and Banks [1]}

I present the voting model of Austen-Smith and Banks [1] as a game with incomplete information in the following way: There is a set of $I=\{1,2, \cdots, n\}$ voters, who have to vote over two alternatives; assume that $n$ is odd and $n \geq 3$. Denote by $\Theta=\{A, B\}$ the set of states of nature. Each voter has an action set $V_{i}=\{A, B\}$, in which $A$ means "choose alternative $\mathrm{A}^{\prime \prime}$ and $B$ means "choose alternative B". Before voters simultaneously cast their votes, each receives an independent private signal $t_{i} \in T_{i}=\{a, b\}$. Thus, $T_{i}$ is the type set of voter $i$. The signals are assumed to be conditionally independent given the state of nature. Let $\Omega=\Theta \times T$ be the set of the states of the world, where $T=\times_{i \in I} T_{i}$. Then, let the probability $\pi \in \Delta(\Omega)$ be the common prior, which is the joint probability distribution of the state of nature and types of all voters, such that,

$$
\pi(A)=p, \pi(B)=1-p ;
$$

and

$$
\pi(t, \theta)=\pi(\theta) \pi(t \mid \theta)=\pi(\theta) \underset{i \in I}{\times} r_{\theta}\left(t_{i}\right),
$$


where $t=\left(t_{1}, \ldots, t_{n}\right) \in T$ is the type profile and $r_{\theta} \in \Delta\left(T_{i}\right)$ is the probability of receiving the signal $a$ or $b$ conditional on $\theta \in \Theta$. Therefore, a type profile $t$ is drawn according to the prior $\pi(t)$ over $T$, that is,

$$
\pi(t)=p r_{A}(t)+(1-p) r_{B}(t),
$$

where $r_{A}(a) \in(1 / 2,1)$ and $r_{B}(b) \in(1 / 2,1)$.

Given $k \in \mathbb{N}$ and $k \leq n$, the alternative $A$ is chosen if the number of votes for $\mathrm{A}$, which is defined as $\left|\left\{i: v_{i}=A\right\}\right|$, is greater than or equal to $k$, where $v_{i}$ is the action chosen by voter $i$; the alternative $B$ is chosen otherwise. Formally, define the voting rule $\Lambda_{k}: \times_{i \in I} V_{i} \rightarrow\{A, B\}$, such that

$$
\Lambda_{k}(v)= \begin{cases}A & \text { if }\left|\left\{i: v_{i}=A\right\}\right| \geq k \\ B & \text { if }\left|\left\{i: v_{i}=A\right\}\right|<k\end{cases}
$$

for all action profiles $v$ in $V=\times_{i \in I} V_{i}$. All voters are assumed to have the same ex-ante preferences, specifically $u(A, A)=u(B, B)=1$ and $u(A, B)=u(B, A)=0, \forall i \in I$. This concludes the definition of the voting game of Austen-Smith and Banks [1], which is denoted by $G=\left\langle I, \Theta,\left(T_{i}, V_{i}\right)_{i \in I}, u, \pi\right\rangle$.

A strategy for voter $i \in I$ in $G$ is a function $\sigma_{i}: T_{i} \rightarrow \triangle\left(V_{i}\right)$. I denote $\sigma_{i}\left(v_{i} \mid t_{i}\right)$ as the probability that voter $i$ chooses action $v_{i} \in V_{i}$ when observing $t_{i} \in T_{i}$. A strategy profile, defined as $\sigma=\left(\sigma_{1}, \ldots, \sigma_{n}\right) \in \Sigma$ and $\Sigma=\times_{i \in I} \Sigma_{i}$, is the set of strategy profiles in $G$. As is standard, denote by $\sigma_{-i}$ the strategy profile $\left(\sigma_{j}\right)_{j \in I \backslash\{i\}}$, that is the strategy profile of all voters except $i$. Then, the common ex-ante expected payoff for each voter can be represented as $E u\left(\theta, \Lambda_{k}\left(\sigma_{i}\left(t_{i}\right), \ldots, \sigma_{n}\left(t_{n}\right)\right)\right)$, which is precisely the probability of a correct decision made by $I$ when the strategy profile $\sigma$ is used, given $\theta$ is the true state of the nature.

Given $\sigma \in \Sigma$, denote by $x_{i}\left(\sigma_{i}\right): \Theta \times T_{i} \rightarrow\{0,1\}$ the indicator variable of the set of correct voting of voter $i$, when using strategy $\sigma_{i}$, that is,

$$
x_{i}\left(\sigma_{i} ; \theta, t_{i}\right)= \begin{cases}1 & \text { if }\left(\theta, v_{i}\left(t_{i}\right)\right)=(A, A) \text { or }\left(\theta, v_{i}\left(t_{i}\right)\right)=(B, B) \\ 0 & \text { otherwise. }\end{cases}
$$

Here, with a slight abuse of notation, further denote by $v_{i}\left(t_{i}\right)$ the realised pure action of $i$ with type $t_{i}$, who uses strategy $\sigma_{i}$. Therefore, given $\sigma$, the probability distribution $\pi$ on $\Omega$ induces a joint probability distribution defined over $X=\left\{x=\left(x_{1}, \ldots, x_{n}\right) \mid x_{i} \in\{0,1\}\right\}$, which can be denoted by $\pi_{\sigma}(\theta, t)$. The random variable $f\left(\Lambda_{k}\right): X \rightarrow\{0,1\}$ associated with the given voting rule is

$$
f\left(\Lambda_{k} ; x(\sigma ; \theta, t)\right)= \begin{cases}1 & \text { if }\left(\theta, \Lambda_{k}\left(v_{1}\left(t_{1}\right), \ldots, v_{n}\left(t_{n}\right)\right)\right)=(A, A) \text { or }\left(\theta, \Lambda_{k}\left(v_{1}\left(t_{1}\right), \ldots, v_{n}\left(t_{n}\right)\right)\right)=(B, B) \\ 0 & \text { otherwise. }\end{cases}
$$

The random variable $f$ partitions $X$ into two mutually exclusive and exhaustive sets, $X_{f_{1}}$ and $X_{f_{0}}=X \backslash X_{f_{1}}$, where $X_{f_{1}}=\left\{x \mid f\left(\Lambda_{k} ; x\right)=1\right\}$. Thus, the common ex-ante expected payoff for each voter under the voting rule $\Lambda_{k}$ is given by $E u=\operatorname{Pr}\left(x \in X_{f_{1}}\right)$.

Next, denote by $T_{-i}^{p i v}=\left\{t_{-i} \in T_{-i}|| v_{-i}\left(t_{-i}\right)=A \mid=k-1\right\}$ the type subprofile which, given strategy $\sigma_{-i}$, gives exactly $k-1$ votes of alternative $A$ from all voters except $i$. In addition, denote by $T_{-i, f_{1}}^{\overline{p i v}}=$ $\left\{t_{-i} \in T_{-i}|| v_{-i}\left(t_{-i}\right)=A \mid \neq k-1\right.$ and $\left.f\left(\Lambda_{k} ; x_{-i}\left(\sigma_{-i} ; \theta, t_{-i}\right)\right)=1\right\}$ the non-pivotal type subprofile of all voters except $i$, which leads to the collective decisions given strategy $\sigma_{-i}$.

Then, $X_{-i}^{p i v}=\left\{x_{-i}=\left(x_{j}\right)_{j \in I \backslash\{i\}} \mid x_{-i}: \Theta \times T_{-i}^{p i v} \rightarrow\{0,1\}^{-i}\right\}$ is the set of all vectors of the random variables, which is defined over the pivotal voting profiles of all voters except $i$. Furthermore, $X_{-i, f_{1}}^{\overline{p i v}}=$ $\left\{x_{-i} \mid x_{-i} \notin X_{-i}^{p i v}\right.$ and $\left.f\left(\Lambda_{k} ; x_{-i}\right)=1\right\}$ is the set of all vectors of the random variables that indicates the correct selected alternative, defined over the non-pivotal voting profiles of the $I \backslash\{i\}$ voters. 
The ex-ante expected payoff can, thus, be rewritten as

$$
\operatorname{Pr}\left(x \in X_{f_{1}}\right)=\operatorname{Pr}\left(x_{i}=1, x_{-i} \in X_{-i}^{p i v}\right)+\operatorname{Pr}\left(x_{-i} \in X_{f_{1}}^{\overline{p i v}}\right),
$$

and, the interim expected utility of voter $i$ given the private signal $t_{i}$, as

$$
\operatorname{Pr}\left(x \in X_{f_{1}} \mid t_{i}\right)=\operatorname{Pr}\left(x_{i}=1, x_{-i} \in X_{-i}^{p i v} \mid t_{i}\right)+\operatorname{Pr}\left(x_{-i} \in X_{-i, f_{1}}^{\overline{p i v}} \mid t_{i}\right) .
$$

After receiving $t_{i}$, voter $i$ updates their prior belief according to Bayes' rule, thereby forming the posterior belief $\pi^{t_{i}}$ of the joint probability distribution of the states of the world. Next, denote the posterior belief by $\pi^{t_{i}}(\theta, t)=\pi^{t_{i}}(\theta) \times_{i \neq j} r_{\theta}\left(t_{j}\right)$. Then, the interim expected payoff for voter $i$, given strategy profile $\sigma$ can be written as

$$
E u\left[\sigma_{i}, \sigma_{-i} \mid t_{i}\right]=\sum_{\theta \in \Theta} \sigma_{i}\left(\theta \mid t_{i}\right) \sum_{t_{-i} \in T_{-i}^{p i v}} \pi_{\sigma_{-i}}^{t_{i}}\left(\theta, t_{-i}\right)+\sum_{t_{-i} \in T_{-i, f_{1}}^{\overline{p i v}}} \pi_{\sigma_{-i}}^{t_{i}}\left(\theta, t_{-i}\right) .
$$

A Bayesian Nash equilibrium of $G$ is a strategy profile $\sigma^{*}=\left(\sigma_{1}^{*}, \ldots, \sigma_{n}{ }^{*}\right)$ such that for all $i \in I$, $E u\left[\sigma_{i}{ }^{*}, \sigma_{-i}{ }^{*} \mid t_{i}\right] \geq E u\left[\sigma_{i}, \sigma_{-i}{ }^{*} \mid t_{i}\right]$ for every $t_{i} \in T_{i}$ and $\sigma_{i} \in \Sigma_{i}$, where $\sigma_{-i}{ }^{*}=\left(\sigma_{j}^{*}\right)_{j \in I \backslash\{i\}}$. That is for all $\sigma_{i} \in \Sigma_{i}$,

$$
\begin{gathered}
\sum_{\theta \in \Theta} \sigma_{i}^{*}\left(\theta \mid t_{i}\right) \sum_{t_{-i} \in T_{-i}^{p i o}} \pi_{\sigma_{-i}^{*}}^{t_{i}}\left(\theta, t_{-i}\right)+\sum_{t_{-i} \in T_{-i, f_{1}}^{p i v}} \pi_{\sigma_{-i}}^{t_{i}}\left(\theta, t_{-i}\right) \geq \sum_{\theta \in \Theta} \sigma_{i}\left(\theta \mid t_{i}\right) \sum_{t_{-i} \in T_{-i}^{p i o}} \pi_{\sigma_{-i}^{*}}^{t_{i}^{*}}\left(\theta, t_{-i}\right)+\sum_{t_{-i} \in T_{-i, f_{1}}^{p_{p}^{p i o}}} \pi_{\sigma_{-i}^{*}}^{t_{i}}\left(\theta, t_{-i}\right) \\
\sum_{\theta \in \Theta} \sigma_{i}^{*}\left(\theta \mid t_{i}\right) \sum_{t_{-i} \in T_{-i}^{p i o}} \pi_{\sigma_{-i}^{*}}^{t_{i}}\left(\theta, t_{-i}\right) \geq \sum_{\theta \in \Theta} \sigma_{i}\left(\theta \mid t_{i}\right) \sum_{t_{-i} \in T_{-i}^{p i o}} \pi_{\sigma_{-i}^{*}}^{t_{i}}\left(\theta, t_{-i}\right) .
\end{gathered}
$$

Since $\pi\left(\theta, t_{-i}\right)$ is a singleton so is each $\pi^{t_{i}}\left(\theta, t_{-i}\right)$, and hence $\sum_{t_{-i} \in T_{-i, f_{1}}^{\overline{p i v}}} \pi_{\sigma_{-i}^{*}}^{t_{i}}\left(\theta, t_{-i}\right)$ in Equation (1), which implies Equation (2).

I denote by $p i v_{i}$ the event that voter $i$ is pivotal, which is the case when $t_{-i} \in T_{-i}^{\text {piv }}$ given strategy profile $\sigma_{-i}$. Hence, the strategy profile $\sigma^{*}=\left(\sigma_{1}{ }^{*}, \ldots, \sigma_{n}{ }^{*}\right)$ is a Bayesian Nash Equilibrium of the game $G$ if and only if for every $i \in I$ and every $t_{i} \in T_{i}$,

$$
\sigma^{*} \in \underset{\sigma \in \Sigma}{\operatorname{argmax}} \sum_{\theta \in \Theta} \sigma_{i}\left(\theta \mid t_{i}\right) \pi_{\sigma_{-i}}^{t_{i}}\left(\theta, p i v_{i}\right) .
$$

\section{Pivotality in an Ambiguous Voting Model}

In this section, I show the equilibrium condition for an ambiguous voting game, in which all players have a common set of priors $\Pi \subseteq \Delta(\Omega)$, which is a closed and convex set of probability distributions over the states of the world. I only look at the cases where the ambiguity regarding the structure of the game is embedded in the prior distribution. The ambiguity regarding each player's belief about their opponents' strategies is not considered.

The study of Kajii and Ui [14] proves the existence of the mixed strategy equilibrium of an incomplete information game with multiple priors, a tuple $\widehat{G}=\left\langle I, \Theta,\left(T_{i}, V_{i}, \Pi_{i}\right)_{i \in I}, \Phi, u\right\rangle$. In such a game, the incompleteness of the information is expressed by a non-empty compact set of priors $\Pi_{i}$ over a finite set of payoff relevant states, the types $T_{i}$ and the exogenously given updating rule $\Phi$; and the action set $V_{i}$ of each voter is assumed to be finite.

Given multiple priors, voters are no longer standard Bayesian players. For each $t_{i} \in T_{i}$ and $\pi_{i} \in \Pi_{i}$, denote by $\pi_{i}^{t_{i}}=\pi_{i}\left(\cdot \mid t_{i}\right)$ the conditional probability and by $\Pi_{i}^{t_{i}}=\left\{\pi_{i}^{t_{i}} \in \Delta(\Omega) \mid \pi_{i} \in \Pi_{i}\right\}$ the set of conditional probabilities over $\Omega$. An updating rule $\Phi: T_{i} \rightarrow 2^{\Pi_{i}^{t_{i}}}$ indicates that for each $t_{i} \in T_{i}$, there is a non-empty compact subset of $\Pi_{i}^{t_{i}}$, i.e, the set of posterior probabilities $\Phi_{i}^{t_{i}}$. 
The additional assumption is that players adopt the Maxmin decision rule of Gilboa and Schmeidler [10] implying that each player uses the least favourable posterior $\phi_{i}^{t_{i}} \in \Phi_{i}^{t_{i}}$ obtained from $\Phi\left(t_{i}\right)$ to evaluate their actions.

Therefore, a strategy profile $\sigma^{*}=\left(\sigma_{1}{ }^{*}, \ldots, \sigma_{n}{ }^{*}\right)$ is a Nash Equilibrium of the game $\widehat{G}$ if and only if for every $i \in I$ and every $t_{i} \in T_{i}$,

$$
\min _{\phi_{i}^{t_{i}} \in \Phi_{i}^{t_{i}}} E \mathcal{U}\left(\sigma_{i}^{*}\left(t_{i}\right), \sigma_{-i}{ }^{*} \mid t_{i}\right) \geq \min _{\phi_{i}^{t_{i}} \in \Phi_{i}^{t_{i}}} E u\left(\sigma_{i}\left(t_{i}\right), \sigma_{-i}{ }^{*} \mid t_{i}\right),
$$

for all $\sigma_{i} \in \Sigma_{i}$.

The voting game with a common set of priors is a special case of the game $\widehat{G}$ proposed by Kajii and Ui [14]. In the proposed ambiguous voting game, the closed and convex set of priors are assumed to be common. In addition, the updating rule adopted by all Maxmin voters is the Full Bayesian Updating rule of Pires [15]. These two assumptions leads to the fact that $\Phi^{t_{i}}=\Pi^{t_{i}}$.

Hence, for the voting game $\tilde{G}=\left\langle I, \Theta,\left(T_{i}, V_{i}\right)_{i \in I}, \Pi, \Phi, u\right\rangle, \sigma^{*}=\left(\sigma_{1}{ }^{*}, \ldots, \sigma_{n}{ }^{*}\right)$ is a Nash Equilibrium if and only if for every $i \in I$ and every $t_{i} \in T_{i}$,

$$
\sigma^{*} \in \underset{\sigma \in \Sigma}{\operatorname{argmax}} \min _{\pi^{t_{i}} \in \Pi^{t_{i}}} E u\left(\sigma_{i}\left(t_{i}\right), \sigma_{-i} \mid t_{i}\right) .
$$

Next, I use the voting model of Ellis [8], which assumes ambiguity in the probability distribution over the states of the nature, and the voting model of Fabrizi et al. [9] ${ }^{1}$, which assumes the ambiguous likelihood of the type distribution, as examples to prove the insufficiency of conditioning only on pivotality to establish the equilibrium condition for ambiguous voting games.

\section{An Illustration: Ellis [8]}

In the voting model of Ellis [8], there is a common set of priors $\Pi$, which is a closed and convex set of probability distributions over the states of the world, because the probability distribution of the state of nature is assumed to be ambiguous. In particular, the marginal probability of the state of nature $A$, denoted by

$$
\pi(A):=\sum_{t \in T} \pi(A, t),
$$

is between $\underline{p}$ and $\bar{p}$, where $0<\underline{p} \leq \bar{p}<1$, and

$$
\pi(\theta, t)=\underset{i \in I}{X} \pi\left(\theta, t_{i}\right)=\underset{i \in I}{X}\left[\pi\left(t_{j} \mid \theta\right) \pi(\theta)\right]=\pi(\theta) \underset{i \in I}{X_{\theta}}\left(t_{i}\right),
$$

for all $(\theta, t) \in \Omega$. Thus, for every $p \in[\underline{p}, \bar{p}]$, there exists

$$
\pi(A, t)=p \underset{i \in I}{\times} r_{A}\left(t_{i}\right) \text { and } \pi(B, t)=(1-p) \underset{i \in I}{X_{B}} r_{B}\left(t_{i}\right)
$$

Upon receiving $t_{i}$, voter $i$ forms a set of posteriors $\Pi\left(\cdot \mid t_{i}\right)$ by updating each measure in $\Pi$. According to the updating rule $\Phi$, the posterior probability $\pi^{t_{i}}(A)$ is between $\underline{p}^{t_{i}}$ and $\bar{p}^{t_{i}}$ so that

$$
\pi^{t_{i}}(A, t)=p^{t_{i}} \underset{i \neq j}{\times} r_{A}\left(t_{j}\right) \text { and } \pi^{t_{i}}(B, t)=\left(1-p^{t_{i}}\right) \underset{\substack{i \neq j \\ r_{B}}}{\left(t_{j}\right)},
$$

for every $p^{t_{i}} \in\left[p^{t_{i}}, \bar{p}^{t_{i}}\right]$.

In addition, the voters have Maxmin preferences, which means that voter $i$ evaluates strategy $\sigma_{i}\left(t_{i}\right)$ by the minimum probability of selecting the correct alternative.

1 An earlier version of the paper by Fabrizi et al. [9] was circulated with the title "The good, the bad, and the not so ugly: Unanimity voting with ambiguous information". 
Thus, the ambiguous voting game of Ellis [8] is defined by the game $\tilde{G}=\left\langle I, \Theta,\left(T_{i}, V_{i}\right)_{i \in I}, \Pi, \Phi, u\right\rangle$. The interim expected payoff to a strategy $\sigma_{i}$ after $t_{i} \in T_{i}$, given $\sigma_{-i} \in \Sigma_{-i}$ is

$$
\begin{aligned}
U\left(\sigma_{i}\left(t_{i}\right), \sigma_{-i} \mid t_{i}\right) & =\min _{\pi^{t_{i}} \in \Pi^{t_{i}}} E u\left(\sigma_{i}\left(t_{i}\right), \sigma_{-i} \mid t_{i}\right) \\
& =\min _{\pi^{t_{i} \in \Pi^{t_{i}}}} \sum_{\theta \in \Theta} \sigma_{i}\left(\theta \mid t_{i}\right) \sum_{t_{-i} \in T_{-i}^{p i v}} \pi_{\sigma_{-i}}^{t_{i}}\left(\theta, t_{-i}\right)+\sum_{t_{-i} \in T_{-i, f_{1}}^{\overline{p i v}}} \pi_{\sigma_{-i}}^{t_{i}}\left(\theta, t_{-i}\right) \\
& =\min _{\pi^{t_{i}}(\theta) \in \Pi^{i}(\theta)} \sum_{\theta \in \Theta} \pi^{t_{i}}(\theta)\left[\sigma_{i}\left(\theta \mid t_{i}\right) \sum_{t_{-i} \in T_{-i}^{p i v}} \pi_{\sigma_{-i}}^{t_{i}}\left(t_{-i}\right)+\sum_{t_{-i} \in T_{-i, f_{1}}^{\overline{p i v}}} \pi_{\sigma_{-i}}^{t_{i}}\left(t_{-i}\right)\right] \cdot X
\end{aligned}
$$

Then, for every $t_{i} \in T_{i}$ and every $\sigma_{i} \in \Sigma_{i}$,

$$
U\left(\sigma_{i}^{*}\left(t_{i}\right), \sigma_{-i}^{*} \mid t_{i}\right) \geq U\left(\sigma_{i}\left(t_{i}\right), \sigma_{-i}^{*} \mid t_{i}\right)
$$

if and only if

$$
\begin{aligned}
\min _{\pi_{i}^{t_{i}}(\theta) \in \Pi^{t_{i}}(\theta)} \sum_{\theta \in \Theta} \pi^{t_{i}}(\theta)\left[\sigma_{i}^{*}\left(\theta \mid t_{i}\right) \sum_{t_{-i} \in T_{-i}^{p i o}} \pi_{\sigma_{-i}^{*}}^{t_{i}}\left(t_{-i}\right)+\sum_{t_{-i} \in T_{-i, f_{1}}^{\overline{p i o}}} \pi_{\sigma_{-i}^{*}}^{t_{i}}\left(t_{-i}\right)\right] \\
\geq \min _{\pi^{t_{i}}(\theta) \in \Pi^{t_{i}}(\theta)} \sum_{\theta \in \Theta} \pi^{t_{i}}(\theta)\left[\sigma_{i}\left(\theta \mid t_{i}\right) \sum_{t_{-i} \in T_{-i}^{p i v}} \pi_{\sigma_{-i}^{*}}^{t_{i}^{*}}\left(t_{-i}\right)+\sum_{t_{-i} \in T_{-i, f_{1}}^{p^{i p o}}} \pi_{\sigma_{-i}^{*}}^{t_{i}^{*}}\left(t_{-i}\right)\right] .
\end{aligned}
$$

In Equation (3), as $\sigma_{i}{ }^{*} \neq \sigma_{i}$, the posterior $\pi^{t_{i}}(\theta)$ which gives the minimum expected utility of choosing strategy $\sigma_{i}{ }^{*}$ might not necessarily be the one that leads to the minimum expected utility of choosing strategy $\sigma_{i}$. This simply shows that the probability that the correct alternative gets selected in the non-pivotal scenario cannot cancel out for this game $\tilde{G}$.

I denote by $\overline{p i v}_{f_{1}}$ the event in which the correct alternative is selected by $I \backslash\{i\}$ voters. Then, Equation (3) implies that

$$
\sigma^{*} \in \underset{\sigma \in \Sigma}{\operatorname{argmax}} \sum_{\pi^{t_{i}}(\theta) \in \Pi^{t_{i}}(\theta)} \sum_{\theta \in \Theta} \pi^{t_{i}}(\theta)\left[\sigma_{i}\left(\theta \mid t_{i}\right) \pi_{\sigma_{-i}}^{t_{i}}(p i v)+\pi_{\sigma_{-i}}^{t_{i}}\left(\overline{p i v}_{f_{1}}\right)\right] .
$$

\section{Another Illustration: Fabrizi et al. [9]}

Another variation of the voting model is to assume that the probability distribution of the types conditional on the state of the nature is ambiguous. That is conditional on state $\theta$, the signal that voter $i$ gets is distributed according to one of the distributions in a closed and convex set $R_{\theta}$, such that $r_{A}(a)=r_{B}(b)=\mu \in[\underline{\mu}, \bar{\mu}]$, where $1 / 2<\underline{\mu} \leq \bar{\mu}<1$. This forms a common set of priors $\Pi$ over the states of the world such that $\pi \in \Pi$ if and only if there is a $\mu \in[\underline{\mu}, \bar{\mu}]$ so that $\pi(\theta, t)=\pi(\theta) \times_{i \in I} r_{\theta}\left(t_{i}\right)$, for all $(\theta, t) \in \Omega$. Hence, the voting game by Fabrizi et al. [9] can also be defined by $\tilde{G}$, except that the set of common priors is different from that of Ellis [8]. Consequently, both the pivotal and non-pivotal scenarios should be considered in the equilibrium condition of a voting game with ambiguity regarding the type distributions.

In each of the voting models discussed above, in spite of the different sources of the ambiguity, there exists a closed and convex set of common priors $\Pi$. Because the Full Bayesian Updating rule $\Phi$ is not singleton-valued, so is the set of posteriors $\Pi\left(\cdot \mid t_{i}\right)$. Hence, for Maxmin voters, the posteriors associated with different strategies are not necessarily the same, which precludes the possibility to eliminate the non-pivotal scenarios.

Therefore, a strategy profile $\sigma^{*}$ is an equilibrium if and only if for every voter $i$ of type $t_{i}$, it maximises their expected utility, given the posterior beliefs and that other voters follow the strategy profile $\sigma_{-i}{ }^{*}$. That is, the minimum probability of the correct alternative, which encompasses the probability of voter $i$ being pivotal and choosing the correct alternative, as well as the probability of voter $i$ being non-pivotal, whereas $I \backslash\{i\}$ voters select the correct alternative, is maximised by $\sigma^{*}$. 
I reiterate the main finding in the following proposition.

Proposition 1. For the ambiguous voting game defined by $\tilde{G}=\left\langle I, \Theta,\left(T_{i}, V_{i}\right)_{i \in I}, \Pi, \Phi, u\right\rangle, \sigma^{*}=\left(\sigma_{1}^{*}, \ldots, \sigma_{n}{ }^{*}\right)$ is the Nash Equilibrium if and only if for every $i \in I$ and every $t_{i} \in T_{i}$,

$$
\sigma^{*} \in \underset{\sigma \in \Sigma}{\operatorname{argmax}} \min _{\pi^{t_{i}}(\theta) \in \Pi^{t_{i}}(\theta)} \sum_{\theta \in \Theta} \pi^{t_{i}}(\theta)\left[\sigma_{i}\left(\theta \mid t_{i}\right) \pi_{\sigma_{-i}}^{t_{i}}(\text { piv })+\pi_{\sigma_{-i}}^{t_{i}}\left(\overline{\text { piv }}_{f_{1}}\right)\right] .
$$

\section{Conclusions}

In this note, I provide simple derivations of the equilibrium conditions for voting games such as those of Austen-Smith and Banks [1] with a unique common prior, and Ellis [8] and Fabrizi et al. [9] with a set of common priors. I demonstrate that each voter cannot determine the best response merely based on the pivotal event. The non-pivotal events are also necessary components in the equilibrium condition whenever there is a common set of priors and an updating rule that is not singleton-valued.

The analysis is confined to the case where voters are Maxmin expected utility maximisers. This could be extended beyond the Maxmin preferences, for example, assuming voters are Choquet expected utility maximisers as in Schmeidler [16], who admit non-additive probabilities on the grand state space of the voting game under ambiguity. Extending the analysis of voting under ambiguity to more general classes of voter preferences, such as to CEU maximisers, would have its merits, but assuming CEU preferences would not change the core point of this note. Moreover, a similar decision-theoretical approach à la Eliaz, Ray, and Razin [11] can be applied to the ambiguous voting game to conduct the equilibrium analysis, and to compare choice shifts with the full information equivalence voting equilibrium. However, to address these points is beyond the scope of this note and is left for future research.

Funding: This research was funded by the Royal Society of New Zealand, Marsden Fund Standard Grant number UOA1617.

Acknowledgments: The author thanks Simona Fabrizi, John Hillas, John Panzar, Matthew Ryan, Ronald Stauber and audiences at the Centre for Mathematical Social Science research seminar for helpful comments. The initial idea for this note benefitted from conversations with Clemens Puppe and Arkadii Slinko. The author also thanks two anonymous referees and the editors for very useful comments on this note.

Conflicts of Interest: The author declares no conflict of interest. The funders had no role in the design of the study; in the collection, analyses, or interpretation of data; in the writing of the manuscript, or in the decision to publish the results.

\section{References}

1. Austen-Smith, D.; Banks, J.S. Information aggregation, rationality, and the Condorcet jury theorem. Am. Political Sci. Rev. 1996, 90, 34-45. [CrossRef]

2. Margolis, H. Pivotal voting. J. Theor. Politics 2001, 13, 111-116. [CrossRef]

3. Margolis, H. Game theory and juries: A miraculous result. J. Theor. Politics 2001, 13, 425-435. [CrossRef]

4. Margolis, H. Pivotal voting and the emperor's new clothes. Soc. Choice Welf. 2002, 19, 95-111. [CrossRef]

5. Dietrich, F.; Spiekermann, K. Epistemic democracy with defensible premises. Econ. Philos. 2013, 29, 87-120. [CrossRef]

6. Dietrich, F.; Spiekermann, K. Jury Theorems. Unpublished work, 2016.

7. Feddersen, T.; Pesendorfer, W. Convicting the innocent: The inferiority of unanimous jury verdicts under strategic voting. Am. Political Sci. Rev. 1998, 92, 23-35. [CrossRef]

8. Ellis, A. Condorcet meets Ellsberg. Theor. Econ. 2016, 11, 865-895. [CrossRef]

9. Fabrizi, S.; Lippert, S.; Pan, A.; Ryan, M. Unanimous Jury Voting with an Ambiguous Likelihood; University of Auckland: Auckland, New Zealand, 2019.

10. Gilboa, I.; Schmeidler, D. Maxmin expected utility with non-unique prior. J. Math. Econ. 1989, 18, $141-153$. [CrossRef]

11. Eliaz, K.; Ray, D.; Razin, R. Choice shifts in groups: A decision-theoretic basis. Am. Econ. Rev. 2006, 96, 1321-1332. [CrossRef] 
12. Dillenberger, D.; Raymond, C. On the Consensus Effect. Unpublished work, 2019.

13. Quiggin, J. A theory of anticipated utility. J. Econ. Behav. Organ. 1982, 3, 323-343. [CrossRef]

14. Kajii, A.; Ui, T. Incomplete information games with multiple priors. Jpn. Econ. Rev. 2005, 56, 332-351. [CrossRef]

15. Pires, C.P. A rule for updating ambiguous beliefs. Theory Decis. 2002, 53, 137-152. [CrossRef]

16. Schmeidler, D. Subjective probability and expected utility without additivity. Econometrica 1989, 57, 571-587. [CrossRef]

(C) (C) 2019 by the author. Licensee MDPI, Basel, Switzerland. This article is an open access article distributed under the terms and conditions of the Creative Commons Attribution (CC BY) license (http:/ / creativecommons.org/licenses/by/4.0/). 